I N S T I T U T O

$\mathrm{DE}$

M E D I C I N A

T R O P I C A L

DE

S ÃO PAULO

JOURNAL OF THE SÃO PAULO INSTITUTE OF TROPICAL MEDICINE

(1)Hospital General de México "Dr. Eduardo Liceaga", Departamento de Micologia, Ciudad de México, México

(2)Hospital General de México "Dr. Eduardo Liceaga", Servicio de Dermatología, Ciudad de México, México

${ }^{(3)}$ Hospital General de México "Dr. Eduardo Liceaga", Servicio de Dermatopatología, Ciudad de México, México

Correspondence to: Alexandro Bonifaz Hospital General de México "Dr. Eduardo Liceaga", Departamento de Micología, Servicio de Dermatología, Dr. Balmis, 148, col. Doctores, CP 06726, Ciudad de México, México

Tel/Fax: +52 55 5761-3923.

E-mail: a_bonifaz@yahoo.com.mx

Received: 19 December 2016

Accepted: 12 May 2017
http://dx.doi.org/10.1590/S1678-9946201759055

\section{Two simultaneous mycetomas caused by Fusarium verticillioides and Madurella mycetomatis}

\author{
Alexandro Bonifaz', Mariana Saldaña², Javier Araiza1, Patricia Mercadillo, \\ Andrés Tirado-Sánchez ${ }^{2}$
}

\section{ABSTRACT}

Mycetoma is a chronic granulomatous disease, classified into eumycetoma caused by fungi and actinomycetoma due to aerobic filamentous actinomycetes. Mycetoma can be found in geographic areas near the Tropic of Cancer. Mexico is one of the countries in which actinomycetoma is endemic. We report an extraordinary case of an adult male with double eumycetoma caused by Madurella mycetomatis and Fusarium verticillioides on both feet.

KEYWORDS: Mycetoma. Eumycetoma. Madurella mycetomatis. Fusarium verticillioides.

\section{INTRODUCTION}

Mycetoma is a chronic granulomatous disease associated with a progressive, inflammatory reaction that clinically resembles a tumor-like soft tissue swelling with sinus tract formation draining purulent exudates containing grains ${ }^{1-3}$. It usually develops after traumatic implantation of soil microorganisms into the subcutaneous tissue, where it develops grains or granules.

Clinically, a nodule gradually forms and evolves into a large subcutaneous mass with sinuses draining pus and grains. The triad of a painless subcutaneous mass, multiple sinuses and purulent or seropurulent discharge containing grains is a characteristic feature of mycetoma ${ }^{1,3}$.

The disease mostly affects young adult patients; however, mycetoma has been described in all ages. Common risk groups include farmers, shepherds and workers in an environment of low socio-economic status ${ }^{1,3}$. In more than $80 \%$ of patients, the lower limbs are mostly affected ${ }^{2,4,5}$.

Mycetoma can be classified as eumycetoma or actinomycetoma caused by fungi or aerobic filamentous bacteria, respectively ${ }^{1,4-6}$.

\section{CASE REPORT}

A 63 year-old man, from Azoyú, Guerrero (542 km Southeast from Mexico City), came to our service presenting 10-year nodular lesions with swelling, draining sinuses and crusting in the lower limbs, mainly distributed on the left foot and the right leg. The early lesion was a solitary nodule on the left foot, followed by the appearance of several draining nodules, close to each other. Two years after the first lesion (8 years of evolution), he developed a nodule in his right ankle, extending to the right aspect of the leg. The patient reported some pruritus and pain on palpation of both dermatoses (Figure 1) There was a history of multiple trauma on both feet, without specifying which objects caused the lesions. Mycological studies: 
direct examination ( $\mathrm{KOH} \mathrm{10 \% )} \mathrm{of} \mathrm{the} \mathrm{discharge} \mathrm{revealed}$ black grains in the left foot sample and white grains on the opposite side of the same foot. On culture, both causative agents, Madurella mycetomatis and Fusarium verticillioides (formerly F. moniliforme), were eumycetes. (Figures 2 and 3). Histopathological examination of the left foot biopsy showed grains, thick hyphae, brownish pigment inside; moreover, in the right foot biopsy, plentiful granules, with a pale whitish coloration, presenting eosinophilic appearance in some areas, were reported (Figures 4 and 5). The center of these collections contained colonies of eumycetes. X-ray of the feet reported osteolysis and periostitis. After screening for renal function tests, routine blood count, G-6-PD activity and audiometry, we decided to start itraconazole $400 \mathrm{mg} /$ day for 58 days and then to add dapsone (DDS) $100 \mathrm{mg} / \mathrm{day}$. The patient did not attend the follow-up visits.

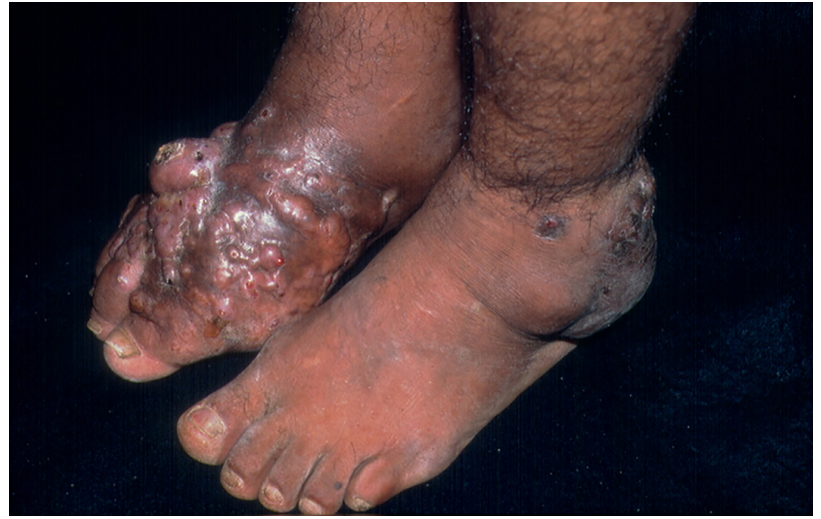

Figure 1 - Left: Fusarium verticilloides mycetoma, Right: Madurella mycetomatis mycetoma

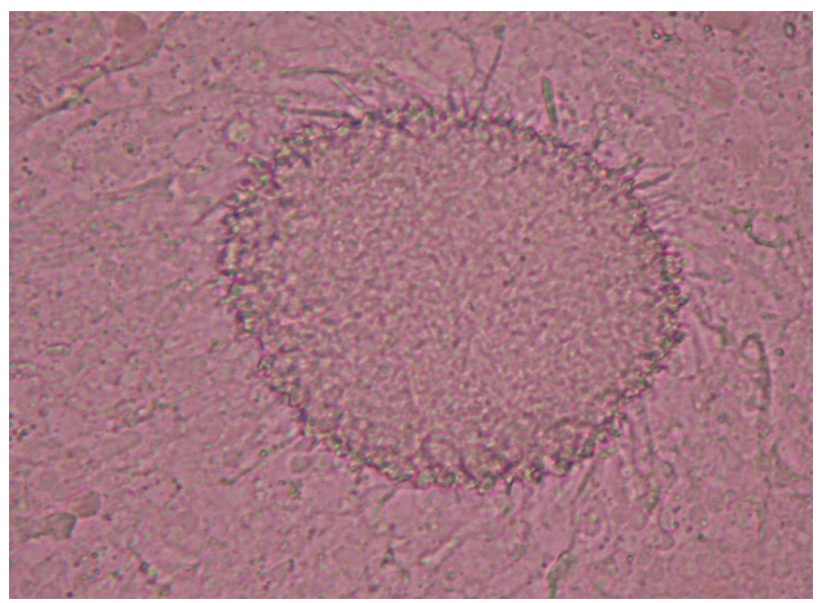

Figure 2 - Direct exam of Fusarium grain (KOH, 10X)

The identification of the fungi was made by traditional methods. As for M. mycetomatis, the following was observed: development of filamentous colony, folded, of brown color, with diffusible ocher pigment. Optimal temperature of growth $37^{\circ} \mathrm{C}$, assimilation of lactose and negative for sucrose.

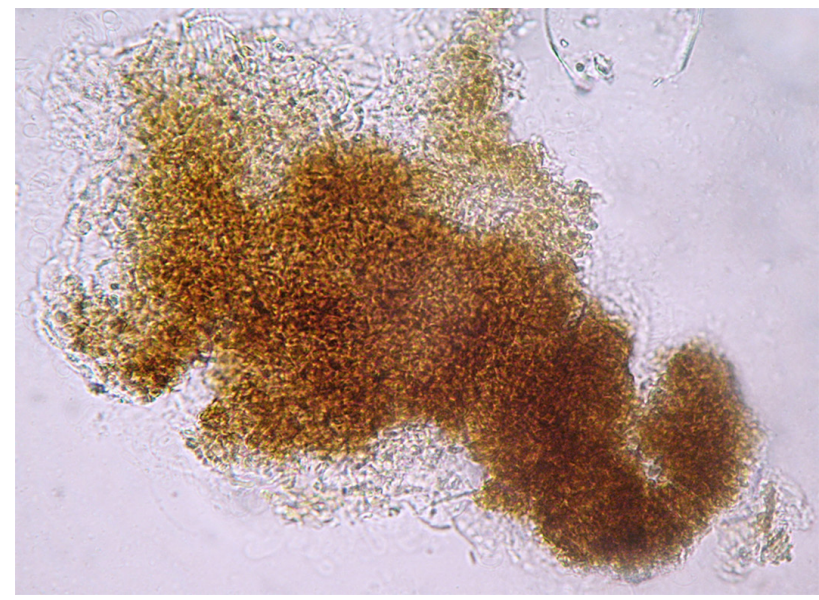

Figure 3 - Direct exam of M. mycetomatis grain (KOH, 10X)

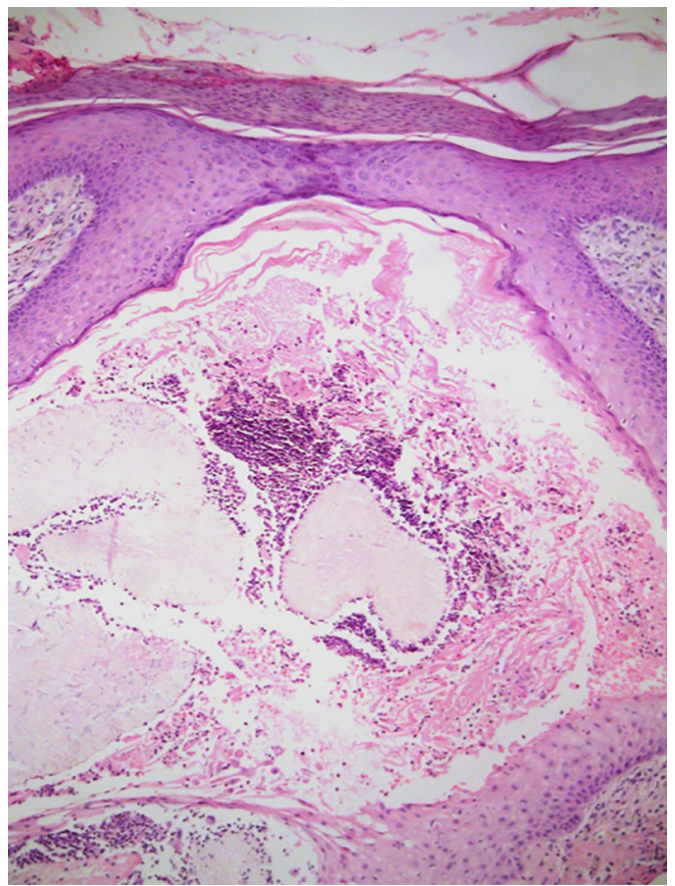

Figure 4 - Suppurative granuloma with a grain of Fusarium (H\&E, 10X)

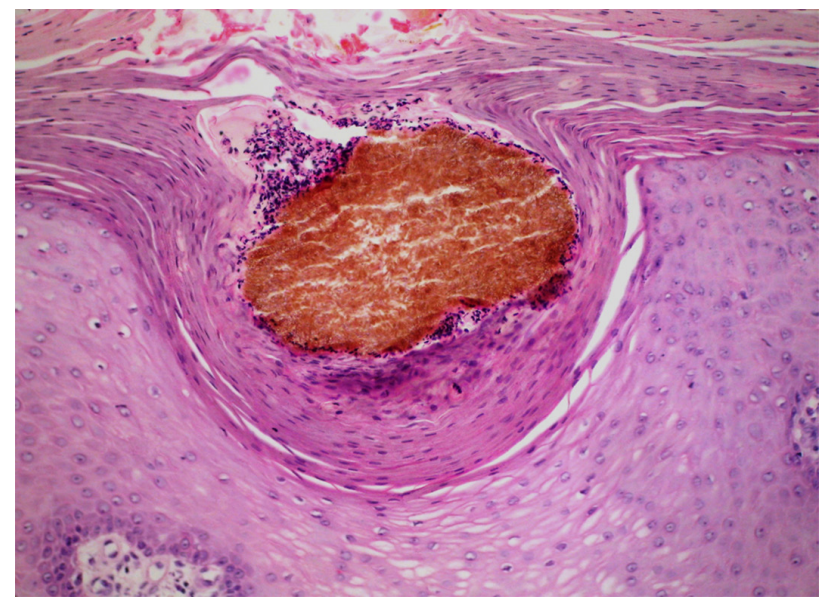

Figure 5 - Black grain of M. mycetomatis (H\&E, $10 \mathrm{X}$ ) 
Microscopically with sterile, melanized mycelium, and formation of scarce sclerotia. F. verticilloides, development of white filamentous colony, fast growing, with diffusible purple pigment. The microscopy showed short conidiophores, with abundant oval microconidia in chains and thin (3-5 septa) and straight macroconidia.

\section{DISCUSSION}

Mycetoma is a chronic, subcutaneous granulomatous disease, beginning after traumatic inoculation of causative microorganisms; it represents a classical neglected disease, most commonly described in people with low socioeconomic level, living in rural regions of Africa, Latin America, and Asia at latitudes classified as "mycetoma belt". This region is located around the Tropic of Cancer, encompassing the countries with the highest rates of infection, including Sudan, Somalia, Senegal, India, Yemen, Mexico, and Venezuela ${ }^{5-7}$. This region is characterized by low humidity and low annual rainfall with well-defined alternating rainy and dry seasons $\mathrm{s}^{2,3,5,6}$.

Eumycetoma is more often seen in tropical and subtropical climates of Africa and Asia, mainly in Sudan and South India. In contrast, actinomycetoma is frequent in North African countries (Morocco, Tunisia). Middle and South American and Asian countries are also endemic regions. In the Western world, mycetomas are diagnosed in immigrants from endemic countries, rarely seen in the native population ${ }^{5,7}$.

Bonifaz et al. ${ }^{4}$, in a study in Mexican patients, showed that eumycetoma represented only $8 \%$ of the mycetomas in this population; melanized fungi was the most commonly isolate. Madurella mycetomatis was the main reported fungi, and it was considered responsible for $25 \%$ of cases worldwide. Some cases have a well-defined history of trauma as a pre-requisite for mycetoma, although there are exceptions ${ }^{8}$.

Fusarium spp. are cosmopolitan fungi, which are thought to be inoculated into the skin by penetrating trauma ${ }^{4,7}$. In Mexico, about 10 cases of mycetoma due to Fusarium species have been reported: 5 by Fusarium solani; 1 by Fusarium subglutinans and 2 by Fusarium sp. Recently we reported two cases related to Fusarium keratoplasticum and Fusarium pseudensiforme, both species belonging to $F$. solani-comple ${ }^{4,8-11} . F$. verticillioides is a common maize phytopathogen, which is the most cultivated plant in Mexico.

Simultaneous double mycetoma is a rare presentation. To our knowledge, Lavalle et al. ${ }^{11}$, in 1987, reported the only case worldwide related to Fusarium sp. and Acremonium sp. Moreover, Fahal et al. ${ }^{3}$, in a study in Sudan, stated that approximately $2 \%$ of affected patients had multiple mycetoma.
A significant symptom of a mycetoma is the painless tumor-like swelling of the subcutaneous tissue, clinically featured as a tumefaction. Contrary to eumycetoma, there is development of grains, representing microcolonies of the causative organism in vivo in the vital tissue. Fungal elements are arranged in the subcutaneous tissue as dense, circumscribed, and radial conglomerates of hyphae enclosed in an amorphous matrix substance. Black grains are characteristic features of Madurella mycetomatis infection. Pale stained (or unstained) grains are characteristic of infections due to Fusarium spp. ${ }^{1,4,10}$.

Relative to the presented case, clinically, it is very similar to other eumycetomas, located on the feet and with the classic morphology. The patient did not present any alteration of the immunity and the fact that he belonged to low socioeconomic levels and had chronic malnutrition aside from precarious working conditions have probably favored the development of mycetoma ${ }^{3,6,8}$.

The next step to achieve diagnosis is the histopathological examination. The clue for diagnosis is epithelioid cell granulomas with grains. Broad, pink-stained hyphae are often seen, surrounded by a sharp basophilic strand. Granulomatous reaction as well as epithelioid cell hyperplasia and multiple nucleus giant cells may develop as a result of a foreign body reaction to fungi in eumycetomas ${ }^{2,4,9}$.

The current therapy for eumycetomas, in tropical and subtropical countries, is the combination of antifungal agents with surgical procedures. Triazole antifungal therapy is the first-line therapy for eumycetomas. The effective dosage of itraconazole for eumycetomas is $400 \mathrm{mg}$ per day, divided into two doses of $200 \mathrm{mg}$. In infections due to Madurella mycetomatis, oral itraconazole 200-400 mg daily is usually useful. Alternatively, terbinafine $250-500 \mathrm{mg}$ once per day might lead to the improvement of eumycetoma. Both therapies are indicated for an extended time, which may be up to one and a half year ${ }^{1,2,4}$.

\section{CONCLUSION}

We present an exceptional case of two eumycetomas in the same patient, one caused by a hyaline fungus (Fusarium verticillioides) and another caused by a melanized fungus (Madurella mycetomatis). This is an example that, in areas of high endemic mycetoma, all types of manifestations can be present.

\section{REFERENCES}

1. Fahal AH. Mycetoma: a thorn in the flesh. Trans R Soc Trop Med Hyg. 2004;98:3-11 
2. Nenoff P, van de Sande WW, Fahal AH, Reinel D, Schofer H. Eumycetoma and actinomycetoma: an update on causative agents, epidemiology, pathogenesis, diagnostics and therapy. J Eur Acad Dermatol Venereol. 2015;29:1873-83.

3. Fahal A, Mahgoub El S, El Hassan AM, Abdel-Rahman ME. Mycetoma in the Sudan: an update from the Mycetoma Research Centre, University of Khartoum, Sudan. PLoS Negl Trop Dis. 2015;9:e0003679.

4. Bonifaz A, Tirado-Sánchez A, Calderón L, Saúl A, Araiza J, Hernández M, et al. Mycetoma: experience of 482 cases in a single center in Mexico. PLoS Negl Trop Dis 2014;8:e3102.

5. van de Sande WW. Global burden of human mycetoma: a systematic review and meta-analysis. PLoS Negl Trop Dis. 2013;7:e2550

6. Carrasco-Zuber JE, Navarrete-Dechent C, Bonifaz A, Fich F, VialLetelier V, Berroeta-Mauriziano D. Cutaneous involvement in the deep mycoses: a literature review. Part I-Subcutaneous mycoses. Actas Dermosifiliogr. 2016;107:806-15.
7. Castro LG, Piquero-Casals J. Clinical and mycologic findings and therapeutic outcome of 27 mycetoma patients from São Paulo, Brazil. Int J Dermatol. 2008;47:160-3

8. López-Martínez R, Méndez-Tovar LJ, Bonifaz A, Arenas R, Mayorga J, Welsh O, et al. Actualización de la epidemiología del micetoma en México. Revisión de 3,933 casos. Gac Med Mex. 2013;149:586-92.

9. Campos-Macías P, Arenas-Guzmán R, Hernadez-Hernandez F. Fusarium subglutinans: a new eumycetoma agent. Med Mycol Case Rep. 2013;2:128-31

10. Al-Hatmi AM, Bonifaz A, Tirado-Sánchez A, Meis JF, de Hoog GS, Ahmed SA. Fusarium species causing eumycetoma: report of two cases and comprehensive review of the literature. Mycoses. 2017;60:242-12.

11. Buot, G, Lavalle P, Mariat F, Suchil P. Etude épidémiologique des mycétomes au Mexique. A propos de 502 cas. Bull Soc Pathol Exot Filiales. 1987;80:329-39. 